\title{
Thoracic aortic aneurysm
}

\section{Background}

An aortic aneurysm is an abnormal widening or ballooning of a section of the aorta, which is the main artery supplying oxygen-rich blood from the heart to the body's organs and tissues. This may occur in the upper part of the aorta in the chest, known as a thoracic aortic aneurysm, or in the lower part of the aorta in the abdomen, known as an abdominal aortic aneurysm. Since they represent a weakened area of the aortic wall, they are susceptible to expansion, tearing or dissection within the wall and ultimately rupture, which may cause significant bleeding and death. Early detection, surveillance and management are critical in preventing such complications of this life-threatening condition.

\section{Risk factors}

There are a number of factors that may contribute to weaken a portion of the aortic wall and increase an individual's risk of developing an aneurysm. These include older age, smoking and high blood pressure. The accumulation of arterial fatty plaques, known as atherosclerosis, also damages and weakens the inner surface of the aorta, which predisposes the aorta to aneurysm formation. In addition, there are genetic conditions, such as Marfan syndrome and Ehlers-Danlos syndrome, where there is a defect in the structural support of the aortic wall, resulting in an increased risk of developing an aneurysm.

\section{Signs and symptoms}

Thoracic aortic aneurysms may expand slowly over time without causing any symptoms. If they become large enough, they may occasionally cause pain in the chest or back, and may exert pressure on nearby structures of the upper airway, causing cough, hoarseness of voice or shortness of breath. If the aneurysm ruptures or causes a dissection within the aortic wall, individuals typically experience a sudden onset of severe, tearing chest pain, which may spread to the neck, jaw or back. Individuals may also experience sudden difficulty breathing, loss of consciousness and signs of stroke, such as weakness and paralysis on one side of the body.

\section{Diagnosis}

Thoracic aortic aneurysms are often detected incidentally from imaging tests carried out for other reasons. If there is a high risk of aneurysm development based on risk factors or if symptoms are present, a number of chest imaging tests can be performed. These include chest $\mathrm{X}$-ray, echocardiography, computed tomography (CT) and magnetic resonance imaging (MRI) of the chest.

\section{Management}

Depending on the symptoms, size and rate of growth of a thoracic aortic aneurysm, treatment may vary from conservative surveillance and medical management to surgical procedures. These all aim to prevent life-threatening rupture of the aneurysm and the need for emergency surgery, which carries much higher rates of mortality and other severe complications. Conservative management involves regular follow-up imaging to monitor growth of the aneurysm and medications aimed at alleviating the effect of risk factors, such as antihypertensive medications to lower blood pressure and statins to reduce cholesterol and the progression of atherosclerosis. For aneurysms that are large, causing symptoms or at increased risk of rupture for any other reason, surgery is indicated. This may be performed through open chest surgery, which typically involves removal of the aneurysm and replacement with a synthetic tube. Some aneurysm repairs may also be carried out by an endovascular approach, whereby a synthetic metal-supported stent-graft attached to a thin tube is inserted through an artery in the leg, guided up to the aorta and fastened in place at the site of the aneurysm. This is aimed at preventing aneurysmal rupture by directing blood flow through the graft and away from the wall of the aneurysm. As always, the risks should be weighed against the benefits for each of these options and discussed with your surgeon.

For more information, please visit the following websites: http://www.mayoclinic.org/diseases-conditions/thoracicaortic-aneurysm/home/ovc-20122021

https://vascular.org/patient-resources/vascular-conditions/ thoracic-aortic-aneurysm http://my.clevelandclinic.org/services/heart/disorders/ aortic-aneurysm/thoracic-aortic-aneurysm

doi: 10.21037/acs.2016.07.05

\begin{tabular}{ll} 
doi: 10.21037/acs.2016.07.05 & $\begin{array}{l}\text { For specific information concerning your medical condition, } \\
\text { ACS suggests that you consult your physician. This page may be } \\
\text { photocopied non-commercially by physicians to share with patients. }\end{array}$ \\
\hline Illustration Editor: Beth Croce & Section Editor: Christopher Cao
\end{tabular}

